\title{
Uniqueness in the determination of loads in multi-span beams and plates
}

\author{
Alexandre Kawano ${ }^{1}$ and Antonino Morassi ${ }^{2}$ \\ ${ }^{1}$ Escola Politecnica, University of Sao Paulo, Brazil \\ email: akawano@usp.br \\ 2 Polytechnic Department of Engineering and Architecture, \\ Università degli Studi di Udine, Italy
}

(Received 19 December 2017)

\begin{abstract}
Most of the results available on the inverse problem of determining loads acting on elastic beams or plates under transverse vibration refer to single beam or single plate. In this paper we consider the determination of sources in multi-span systems obtained by connecting either two Euler-Bernoulli elastic beams or two rectangular Kirchhoff-Love elastic plates. The material of the structure is assumed to be homogeneous and isotropic. The transverse load is of the form $g(t) f(\mathbf{x})$, where $g(t)$ is a known function of time and $f(\mathbf{x})$ is the unknown term depending on the position variable $\mathbf{x}$. Under slight a priori assumptions, we prove a uniqueness result for $f(\mathbf{x})$ in terms of observations of the dynamic response taken at interior points of the structure in an arbitrary small interval of time. A numerical implementation of the method is included to show the possible application of the results in the practical identification of the source term.
\end{abstract}

Keywords: Mechanics of deformable bodies, Euler-Bernoulli equation, Kirchhoff-Love plate equation, Inverse problems.

\section{Introduction}

The identification of forcing terms in beams and plates by measurements of the dynamic response has attracted increasing interest in the inverse problems community in the last years, both from the theoretical and applied point of view. Applications may concern, for example, the determination of the spatial support and magnitude of the contact force produced during impact of objects or the determination of the time-history of wind pressure on exposed surfaces.

It is not easy to draw a complete bibliographic overview on this topic. Here, we limit ourselves to mention some of the contributions from which the interested reader can certainly obtain more information. We recall that the typical mechanical model considered in the literature is either a single beam element or a single rectangular plate under infinitesimal transverse vibration. Here we use the word infinitesimal to designate transverse movements that are small enough so that the linear theory of beams and plates give a good approximation to the behavior of more complex models. The forcing term is usually taken as $g(t) f(\mathbf{x})$, where $g=g(t)$ is a known function of time and $f=f(\mathbf{x})$ is the unknown spatial term depending on the position variable $\mathbf{x}$. It should be noted 
that this decomposition in spatial and temporal part of the acting load can be found in various engineering contexts and in the corresponding code design requirements. Among other applications, we recall the case of blast loading, in which the actions exerted on an exposed structure are modeled as the product between an impulsive (e.g., short duration) function on the time variable and an unknown spatial distribution [1]. Similar load decomposition is also frequently used in ship loading modeling [2]. The material is assumed to be homogeneous and isotropic, and the dynamic response is determined within the framework of the Euler-Bernoulli or Kirchhoff-Love theory for slender beams and thin plates, respectively.

Presentations of the identification problem for single beams, with comprehensive lists of articles can be found in [3]. For plates, Yamamoto [4] proved that the observation of the response of a simply supported rectangular plate on a small segment inside the plate during a sufficient long interval of time is enough to determine uniquely $f$. Extensions to similar problems for a beam equation were also provided in [4]. Along this line of research, it was recently shown in [5] that identification of source terms in plates is also possible when observing a part of the boundary, even for arbitrary small intervals of time. Nicaise and Zaïr [6] considered the determination of the spatial term $f$ given by a linear combination of an unknown finite number of Dirac deltas (e.g., point sources), with both unknown support and amplitude, in a uniform clamped-clamped beam in bending vibration. The authors proved that the measurement of the curvature of the transverse displacement at one end of the beam in an arbitrarily small time of observation determines uniquely $f$. Conditional stability and reconstruction schemes were provided in the paper. An extension of the above results to the unique and conditionally stable determination of point sources in a clamped rectangular plate from the observation of the Laplacian of the transverse displacement at a suitable subset of the boundary of the plate, for a sufficiently large time of observation, was obtained by Zaïr [7]. A vibrating plate with lower order terms and with more regular spatial forcing term $f$ (e.g., $f \in H_{0}^{1}(\Omega)$, where $\Omega$ is the mid-plane of the plate) was considered by Wang [8]. Assuming that both the transverse displacement and its Laplacian vanish on the boundary of the plate, the author found conditions for the unique determination of $f$ from the boundary observation of the normal derivative of both the transverse displacement and of its Laplacian on a suitable subset of the boundary of the plate for an arbitrarily small time of observation. An extension of the results by [8] was provided by Alves et al. [9]. By using the same data as in [8], Alves et al. established the identifiability for a source term given by $\sum_{j=1}^{N} g_{j}(t) \delta_{\xi_{j}}$, where $g_{j}(t)$ are suitable (but unknown) functions of time and $\delta_{\xi_{j}}$ are Dirac deltas with support $\xi_{j} \in \Omega, j=1, \ldots, N, N$ unknown. Suitable extensions to measurements taken in the interior of the plate and conditional stability results were also provided in [9].

All the previous results refer to the inverse problem of identifying a source term in either a single beam or in a single plate. In the construction of modern mechanical and civil engineering structures, single beams or rectangular plates are connected to obtain continuous multi-span beams or multi-span plates placed over intermediate point (beams) or line (plates) supports. In spite of their importance, to our knowledge, studies on source identification for this class of structures are rare. It should be also noticed that the analysis of this inverse problem is not only important per se, but it will provide a theoretical foundation to other identification methods, such as the Statistical Energy 


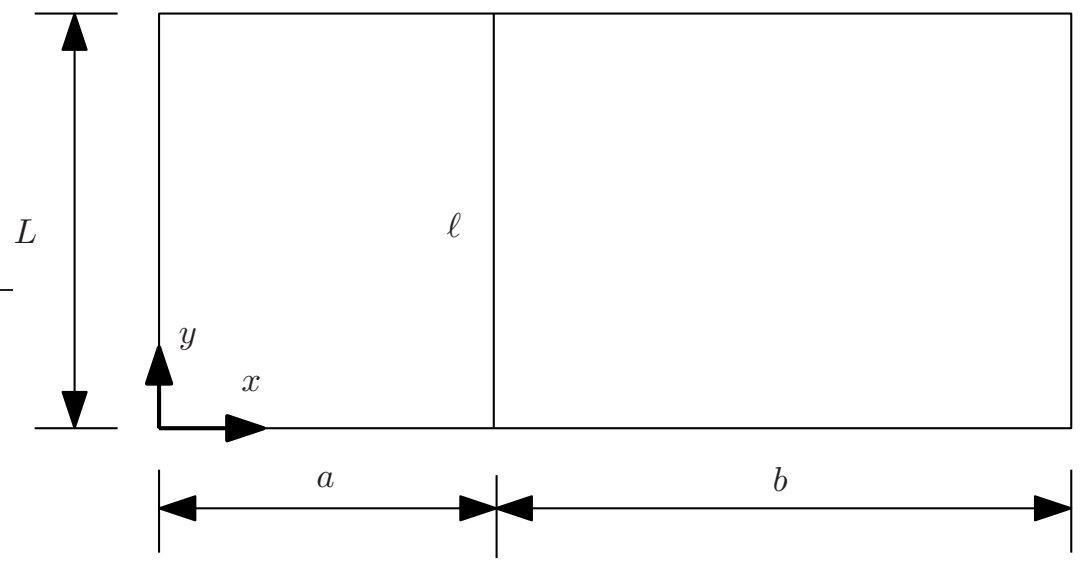

(a)

Figure 1. Two-span plate simply supported at the boundary and with an interior line support.

Method, which are used to locate vibration sources in complex structures by the flow energy balance among their components and by measurements taken on few accessible sites only. For the sake of completeness, we recall that Nicaise and Zair have considered in [10] the problem of determining point sources in a mechanical system obtained by the connection of a finite number of taut strings from Neumann boundary observations taken on a part of the boundary of the system. However, the analysis of string-like structures involves second order differential operators in the space variable rather then fourth order as in the case of beams and plates. We are unaware of analogous results on multi-span beams or plates, and the present research is a contribution to this issue.

In this paper we consider a system composed either by two connected beams or by two connected rectangular plates. In spite of its simplicity, this multi-span system retains all the essence of the problem of transmission of information in the form of waves across an internal - point or line - support for the unique identification of loads from measurements of the vibratory response. It will be clear from the subsequent analysis how the proofs can be extended to multi-span beams and plates formed by any finite number of spans.

In order to present our main result, in this introduction we will refer to the plate system formed by two rectangular plates of dimensions $a \times L$ and $b \times L$, and constant thickness, shown in Figure 1. The plates are perfectly connected along the internal line support $\gamma=\left\{(x, y) \in \mathbb{R}^{2} \mid x=a, 0 \leq y \leq L\right\}$, and the boundary $\partial \Omega$ of the whole multi-span plate $\Omega=(0, a+b) \times(0, L)$ is simply supported, that is the transversal displacement of the plate and its Laplacian vanish on $\partial \Omega$. As mentioned at the beginning of this section, the material is assumed to be homogeneous, linearly elastic and isotropic. The infinitesimal transverse vibrations under the load $g(t) f(x, y)$, where $g \in \mathcal{C}^{1}\left(\left[0, T_{0}\right]\right)$ is a given function of time and $f \in H^{-1}(\Omega \backslash \gamma)$ is a function to be determined, are described within the Kirchhoff-Love theory of thin plates.

The boundary conditions imposed on $\partial \Omega$ prevent to obtain enough information about the unknown spatial source term $f$ from measurements taken at the boundary. Therefore, the inverse problem of determining $f$ is formulated in terms of internal observations. Un- 
der the additional assumption $g(0) \neq 0$, we prove that the knowledge of the time-history of the transverse displacement of the plate measured in an arbitrarily small neighborhood of the line $\left\{\left(x_{0}, y\right), 0 \leq y \leq L\right\}$, where $x_{0}$ is any point of the set $(0, a) \cup(a, a+b)$, for an arbitrarily small registration time, is enough for the unique determination of the function $f$. We refer to Theorem 2.1 for a precise statement and for the corresponding result for a multi-span beam system. The method used to prove uniqueness is essentially based on the series representation of the dynamic response of the structure on its eigenfunction basis, and on the analysis of the almost periodic distribution that arises from it. This idea was originally used by Kawano in [11] to prove that the measurement of the dynamic response of a rectangular plate on a line segment, having arbitrary length and parallel to one of the sides of the plate, is enough for the unique determination of the spatial load distribution, provided that the time-interval of observation is long enough.

The paper is organized as follows. The formulation of the inverse problem and the main uniqueness result (Theorem 2.1) are presented in Section 2. Section 3 is devoted to the analysis of the dynamic response of the multi-span beam or plate system via eigenfunction series representation. A proof of Theorem 2.1 is presented in Section 4. A numerical implementation of the method is shown in Section 5.

\section{Main result}

The first model that we shall consider is a thin Euler-Bernoulli beam made by homogeneous linearly elastic isotropic material. The beam has two spans of length $a$ and $b$, it is supported at the ends $x=0, x=a+b$ and also at the point $x=a$ separating the two spans. The infinitesimal, undamped, transverse vibrations $u=u(x, t)$ under the external transverse force $g(t) f(x)$ are governed by the problem

$$
\left\{\begin{array}{l}
\frac{\partial^{4} u(x, t)}{\partial x^{4}}+\frac{\partial^{2} u(x, t)}{\partial t^{2}}=g(t) f(x), \quad(x, t) \in\left(\Omega_{1} \cup \Omega_{2}\right) \times\left(0, T_{0}\right), \\
u(0, t)=\frac{\partial^{2} u}{\partial x^{2}}(0, t)=0, \quad t \in\left(0, T_{0}\right) \\
{[[u(a, t)]]=0, \quad t \in\left(0, T_{0}\right),} \\
u(a, t)=0, \quad t \in\left(0, T_{0}\right), \\
{\left[\left[\frac{\partial u}{\partial x}(a, t)\right]\right]=0, \quad t \in\left(0, T_{0}\right),} \\
{\left[\left[\frac{\partial^{2} u}{\partial x^{2}}(a, t)\right]\right]=0, \quad t \in\left(0, T_{0}\right),} \\
u(a+b, t)=\frac{\partial^{2} u}{\partial x^{2}}(a+b, t)=0, \quad t \in\left(0, T_{0}\right) \\
u(x, 0)=\frac{\partial u}{\partial t}(x, 0)=0, \quad x \in \Omega_{1} \cup \Omega_{2},
\end{array}\right.
$$

where $T_{0}>0$ is an arbitrary time, $\Omega_{1}=(0, a)$ and $\Omega_{2}=(a, a+b)$ denote the left and right span, respectively, and $[[u(a, t)]]=\lim _{x \rightarrow a^{+}} u(x, t)-\lim _{x \rightarrow a^{-}} u(x, t)$. In the above equations, for the sake of simplicity, the bending stiffness and the mass per unit length of the beam are assumed to be unitary. We notice that the jump conditions $(2.3)-(2.6)$ state that the two ends of the beam $x=a^{-}$and $x=a^{+}$are perfectly connected at $x=a$, and the beam is simply supported at $x=a$. The support at $x=a$ acts on the beam as a concentrated transverse force $V(t) \delta_{a}$, where $V(t)$ is a unknown function and $\delta_{a}$ is the Dirac's delta with support at $x=a$. 
Under the assumption $g \in C^{1}\left(\left[0, T_{0}\right]\right)$ and $f \in H^{-1}\left(\Omega_{1} \cup \Omega_{2}\right)$, there exists a unique solution $u(x, t)$ to $(2.1)-(2.8)$ and $u \in C^{0}\left(\left[0, T_{0}\right], H^{2}\left(\Omega_{1} \cup \Omega_{2}\right)\right) \cap C^{1}\left(\left[0, T_{0}\right], L^{2}\left(\Omega_{1} \cup \Omega_{2}\right)\right)$.

We shall now introduce the motion problem for the two-span continuous thin elastic plate shown in Figure 1. The plate is formed by two rectangular plates, having midplane $\Omega_{1}=(0, a) \times(0, L)$ and $\Omega_{2}=(a, a+b) \times(0, L)$, perfectly connected along the line $\gamma=\{(x, y) \mid x=a, 0 \leq y \leq b\}$. Let us assume that the material is homogeneous, linearly elastic and isotropic, and that the whole plate is simply supported at the boundary and along the line $\gamma$. Let us denote $\Omega=\overline{\Omega_{1} \cup \Omega_{2}}=\{(x, y) \mid 0 \leq x \leq a+b, 0 \leq y \leq L\}$. Working in the framework of infinitesimal deformations and under the kinematical assumptions of the Kirchhoff-Love theory, the dynamic equilibrium problem under the transverse force $g(t) f(x, y)$ is described by the following boundary value problem

$$
\left\{\begin{array}{l}
\Delta \Delta w(x, y, t)+\frac{\partial^{2} w(x, y, t)}{\partial t^{2}}=g(t) f(x, y), \quad(x, y, t) \in\left(\Omega_{1} \cup \Omega_{2}\right) \times\left(0, T_{0}\right) \\
w=\frac{\partial^{2} w}{\partial \nu^{2}}=0, \quad \text { on } \partial \Omega, t \in\left(0, T_{0}\right) \\
{[[w(a, y, t)]]=0, \quad 0 \leq y \leq L, t \in\left(0, T_{0}\right)} \\
w(a, y, t)=0, \quad 0 \leq y \leq L, t \in\left(0, T_{0}\right) \\
{\left[\left[\frac{\partial w}{\partial x}(a, y, t)\right]\right]=0, \quad 0 \leq y \leq L, t \in\left(0, T_{0}\right)} \\
{\left[\left[\frac{\partial^{2} w}{\partial x^{2}}(a, y, t)\right]\right]=0, \quad 0 \leq y \leq L, t \in\left(0, T_{0}\right)} \\
w(x, y, 0)=\frac{\partial w}{\partial t}(x, y, 0)=0, \quad x \in \Omega_{1} \cup \Omega_{2}
\end{array}\right.
$$

where $w=w(x, y, t)$ is the transverse displacement of the point $(x, y)$ belonging to the mid-plane of the plate at time $t, \nu$ is the unit external normal to $\Omega$ and $T_{0}>0$ is an arbitrary time. In the above equations, $\Delta$ is the Laplacian operator with respect to the spatial variables $x, y$ and for every $y \in[0, L]$ we have defined $[[w(a, y, t)]]=$ $\lim _{x \rightarrow a^{+}, x \in \Omega_{2}} w(x, y, t)-\lim _{x \rightarrow a^{-}, x \in \Omega_{1}} w(x, y, t)$. Moreover, to simplify the notation, we have considered unitary bending stiffness and surface mass density. We recall that the jump conditions (2.11)-(2.13) follow by the continuity of $w$ and its first $x$-partial derivative on the internal support $\gamma$, whereas (2.14) expressed the continuity of the bending moment $M_{x x}=-\left(\frac{\partial^{2} w}{\partial x^{2}}+\sigma \frac{\partial^{2} w}{\partial y^{2}}\right)$ at $\gamma$, where $\sigma$ is the Poisson's coefficient of the material. It should be noticed that, analogously to the beam system, the internal support acts on the plate as a concentrated transverse force $W(y, t) \delta_{a}$ along the segment $\gamma$, where $W(y, t)$ is a unknown function.

Concerning the direct problem (2.9)-(2.15), if $g \in C^{1}\left(\left[0, T_{0}\right]\right)$ and $f \in H^{-1}(\Omega \backslash \gamma)$, then there exists a unique solution $w$ and $w \in C^{0}\left(\left[0, T_{0}\right], H^{2}\left(\Omega_{1} \cup \Omega_{2}\right)\right) \cap C^{1}\left(\left[0, T_{0}\right], L^{2}\left(\Omega_{1} \cup \Omega_{2}\right)\right)$, see also Lemma 2.

Our main result on the determination of sources from measurements of the dynamic response of two-span beams and plates is the following uniqueness theorem.

Theorem 2.1 Under the above assumptions, let $g \in C^{1}\left(\left[0, T_{0}\right]\right)$, with $g(0) \neq 0$, be a given function of time for $T_{0}>0$. Then we have:

(I) (Two-span beam system) The function $f \in H^{-1}\left(\Omega_{1} \cup \Omega_{2}\right)$ in equation (2.1) can be uniquely determined by the data

$$
\Gamma=\left\{\langle u, \varphi\rangle \mid \varphi \in \mathcal{C}_{c}^{\infty}\left((0, T) \times \Omega_{0}\right)\right\},
$$


where $\Omega_{0}$ is a neighborhood of any point $x_{0} \in(0, a) \cup(a, a+b)$, with $\Omega_{0} \subset(0, a) \cup(a, a+b)$.

(II) (Two-span plate system) The function $f \in H^{-1}\left(\Omega_{1} \cup \Omega_{2}\right)$ in equation (2.9) can be uniquely determined by the data (2.16), where $u$ is replaced by $w$ and $\Omega_{0}$ is a neighborhood of any line $\left\{\left(x_{0}, y\right) \mid 0 \leq y \leq L\right\}$, with $x_{0} \in(0, a) \cup(a, a+b)$.

In both cases (I) and (II) the time length $T, 0<T<T_{0}$, is arbitrary small.

It should be noted that Theorem 2.1 guarantees the uniqueness of the spatial force term, provided that the dynamic response of the system is taken on a set in space-time domain, which is arbitrarily small not only in time but also in space, and specifically on an arbitrarily small spatial interval for multi-span beams and an arbitrarily thin spatial strip for multi-span plates.

\section{Dynamic response of the whole structure}

The proof of Theorem 2.1 is based on the series representation of the solution to the problems (2.3)-(2.6) and (2.11)-(2.13) in terms of the normal modes (eigenfunctions) of the system. Therefore, the present section is devoted to the analysis of the free vibration problem for the two-span beam and plate system.

\subsection{Eigenvalue problem}

The infinitesimal, undamped, free bending vibration, of radian frequency $\sqrt{\lambda}$ and amplitude $S=S(x)$, of the two-span beam is governed by the eigenvalue problem

$$
\left\{\begin{array}{l}
S^{\prime \prime \prime \prime}(x)=\lambda S(x), \quad x \in \Omega_{1} \cup \Omega_{2} \\
S(0)=S^{\prime \prime}(0)=0 \\
{[[S(a)]]=0} \\
S(a)=0 \\
{\left[\left[S^{\prime}(a)\right]\right]=0} \\
{\left[\left[S^{\prime \prime}(a)\right]\right]=0} \\
S(a+b)=S^{\prime \prime}(a+b)=0
\end{array}\right.
$$

where $S^{\prime}$ denotes the first derivative of $S$ with respect to $x$. The analogous eigenvalue problem for the two-span plate (2.9)-(2.15) consists in finding the eigenpairs $\{\lambda, S=$ $S(x, y)\}$ of

$$
\left\{\begin{array}{l}
\Delta \Delta S(x, y)=\lambda S(x, y), \quad(x, y) \in\left(\Omega_{1} \cup \Omega_{2}\right), \\
S=\frac{\partial^{2} S}{\partial \nu^{2}}=0, \quad \text { on } \partial \Omega \\
{[[S(a, y)]]=0, \quad 0 \leq y \leq L,} \\
S(a, y)=0, \quad 0 \leq y \leq L, \\
{\left[\left[\frac{\partial S}{\partial x}(a, y)\right]\right]=0, \quad 0 \leq y \leq L} \\
{\left[\left[\frac{\partial^{2} S}{\partial x^{2}}(a, y)\right]\right]=0, \quad 0 \leq y \leq L}
\end{array}\right.
$$

where $S=S(x, y)$ is the amplitude of the transverse vibration of the point $(x, y)$ of the plate. 
Let us consider the space $\mathrm{H}^{2}\left(\Omega_{1} \cup \Omega_{2}\right)$ equipped with the $H^{2}$-norm, that is, $\langle\cdot, \cdot\rangle_{\mathrm{H}^{2}\left(\Omega_{1} \cup \Omega_{2}\right)}=$ $\langle\cdot, \cdot\rangle_{\mathrm{H}^{2}\left(\Omega_{1}\right)}+\langle\cdot, \cdot\rangle_{\mathrm{H}^{2}\left(\Omega_{2}\right)}$, where, to simplify the notation, in the sequel it is understood that $\Omega_{1}=(0, a), \Omega_{2}=(a, a+b)$ and $\Omega_{1}=(0, a) \times(0, L), \Omega_{2}=(a, a+b) \times(0, L)$ for the beam and the plate system, respectively. Moreover, the two-dimensional spatial differential operators $\nabla=\left(\frac{\partial}{\partial x}, \frac{\partial}{\partial y}\right), \Delta=\nabla \cdot \nabla$ used for the plate system simply reduces to $\frac{d}{d x}$ and $\frac{d^{2}}{d x^{2}}$ for the beam problem.

The existence of a solution $S \in \mathrm{H}^{2}\left(\Omega_{1} \cup \Omega_{2}\right) \backslash\{0\}$ of the eigenproblems (3.1)-(3.7), (3.8)(3.13) is proved once we prove the existence of an operator $T: \mathrm{H}^{2}\left(\Omega_{1} \cup \Omega_{2}\right) \rightarrow \mathrm{H}^{2}\left(\Omega_{1} \cup \Omega_{2}\right)$ such that $\Delta^{2} T \psi=\psi$, respectively, when $\psi$ solves the above eigenproblem. It is not difficult to prove that $T: H^{2}\left(\Omega_{1} \cup \Omega_{2}\right) \rightarrow H^{2}\left(\Omega_{1} \cup \Omega_{2}\right)$ in fact exists, and it is selfadjoint and compact. Therefore, from the Spectral Theorem, it follows that there exists an orthonormal Hilbert basis for the space $H^{2}\left(\Omega_{1} \cup \Omega_{2}\right)$ formed by the eigenfunctions $\left\{S_{n}\right\}_{n \geq 1}$ of the problems (3.1)-(3.7), (3.8)-(3.13), with positive eigenvalues $\left\{\lambda_{n}\right\}_{n \geq 1}$, $\lim _{n \rightarrow \infty} \lambda_{n}=\infty$. The same set is also an orthogonal set in $\mathrm{L}^{2}\left(\Omega_{1} \cup \Omega_{2}\right)$, and we can normalize the eigenfunctions so that

$$
\left\|S_{n}\right\|_{\mathrm{L}^{2}\left(\Omega_{1} \cup \Omega_{2}\right)}^{2}=\frac{1}{\lambda_{n}} \quad \text { for every } n \in \mathbb{N} .
$$

Therefore, any element in $\mathrm{L}^{2}\left(\Omega_{1} \cup \Omega_{2}\right)$ can be represented by $\sum_{n=1}^{+\infty} c_{n} \sqrt{\lambda_{n}} S_{n}$, for some $\left\{c_{n}\right\}_{n \geq 1} \subset \ell^{2}$.

The following estimates are valid for the eigenfunctions of both the problems (3.1)(3.7), (3.8)-(3.13).

Proposition 1 Let $\left(\lambda_{n}, S_{n}\right), n \geq 1$, be an eigenpair either of the problem (3.1)-(3.7) or of the problem (3.8)-(3.13). We have

$$
\begin{gathered}
\left\|\triangle S_{n}\right\|_{L^{2}\left(\Omega_{1} \cup \Omega_{2}\right)}^{2}=\lambda_{n}\left\|S_{n}\right\|_{L^{2}\left(\Omega_{1} \cup \Omega_{2}\right)}^{2}, \\
\left\|\nabla S_{n}\right\|_{L^{2}\left(\Omega_{1} \cup \Omega_{2}\right)}^{2} \leq \lambda_{n}^{1 / 2}\left\|S_{n}\right\|_{L^{2}\left(\Omega_{1} \cup \Omega_{2}\right)}^{2} .
\end{gathered}
$$

Proof The identity (3.15) follows easily by multiplying the differential equation of the corresponding eigenvalue problem by $S_{n}$, integrating by parts and using the boundary conditions and the jump conditions on the internal support. The estimate (3.16) can be obtained by integration by parts, and by applying Cauchy-Schwarz inequality and identity (3.15).

Finally, consider the set $X \subset \mathrm{H}^{2}\left(\Omega_{1} \cup \Omega_{2}\right)$ generated by sums of the form

$$
X=\overline{\left\{\sum_{n=1}^{N} \alpha_{n} S_{n}:\left\{\alpha_{n}\right\}_{n \in \mathbb{N}} \in \ell^{2}, N \in \mathbb{N}\right\}} .
$$

The space $X$ is a Hilbert space with the norm

$$
\langle\cdot, \cdot\rangle_{X}=\langle\cdot, \cdot\rangle_{\mathrm{H}^{2}\left(\Omega_{1}\right)}+\langle\cdot, \cdot\rangle_{\mathrm{H}^{2}\left(\Omega_{2}\right)},
$$

where $\langle\cdot, \cdot\rangle_{\mathrm{H}^{2}\left(\Omega_{j}\right)}, j=1,2$, is the norm in the usual Sobolev space $\mathrm{H}^{2}\left(\Omega_{j}\right)$.

In the next two sections we shall deal separately the beam and plate cases. 


\subsection{Beam eigensolutions}

To find a solution of the eigenvalue problem (3.1)-(3.7), we write separately in $\Omega_{1}$ and $\Omega_{2}$ the general solution of (3.1) in the form

$$
S(x)=A \sin (\alpha x)+B \cos (\alpha x)+C \sinh (\alpha x)+D \cosh (\alpha x),
$$

where $\alpha=\sqrt[4]{\lambda} \in \mathbb{R}_{+}$. By imposing the prescribed boundary conditions (3.2) at $x=0$ and the jump conditions (3.3)-(3.4) at $x=0$, the expression of the eigenfunction in $\Omega_{1}$ is

$$
S^{a, \alpha}(x)=A_{a}\left(\sin (\alpha x)-\frac{\sin (\alpha a)}{\sinh (\alpha a)} \sinh (\alpha x)\right),
$$

where $A_{a}, A_{a} \neq 0$, is a constant. The corresponding expression for $S^{b, \alpha}$ in $\Omega_{2}$ can be obtained from (3.18) by making the change of variables $\xi=a+b-x$, so that $\xi=0$ corresponds to $x=a+b$, and the interface between the two beams is located at $\xi=b$, or $x=a$. Finally, to get a non null solution $S$ of (3.1)-(3.6), we impose the remaining two jump conditions (3.5)-(3.6) in the two unknowns $A_{a}$ and $A_{b}, A_{b} \neq 0$, and the eigenvalues $\lambda_{n}=\alpha_{n}^{4}$ are the roots of the frequency equation

$$
\frac{\sin (\alpha(a+b))}{\cos (\alpha(a-b))-\cos (\alpha(a+b))}=\frac{1}{2}\left(\frac{\cosh (\alpha a)}{\sinh (\alpha a)}+\frac{\cosh (\alpha b)}{\sinh (\alpha b)}\right) .
$$

Let us notice that the right hand side converges to 1 as $\alpha \rightarrow \infty$ and, therefore, $\alpha_{n}=$ $\frac{\pi}{2} \frac{1+2 n}{a+b}+r(n)$, with $\lim _{n \rightarrow \infty} r(n)=0$. It follows that $\lambda_{n}=\mathcal{O}\left(n^{4}\right)$ as $n \rightarrow \infty$. As an example, Figure 2 shows the behavior of the left and right hand side of equation (3.19) for $a=1$ and $b=2$. The eigenfunctions of (3.1)-(3.7) are given by

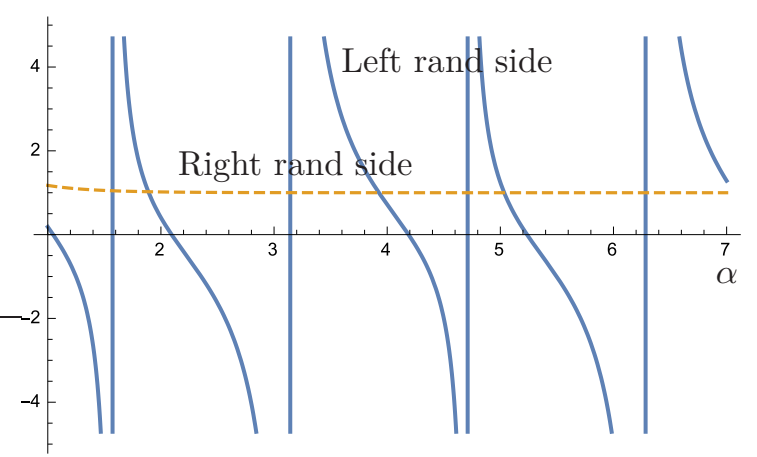

Figure 2. Illustration of the left and right hand side of (3.19)

$$
S_{n}(x)= \begin{cases}S^{a, \sqrt[4]{\lambda_{n}}}(x), & x \in[0, a] \\ S^{b, \sqrt[4]{\lambda_{n}}}(a+b-x), & x \in[a, a+b]\end{cases}
$$

Observation 1 The eigenfunctions of the problem $(3.1)-(3.7)$ belong to $\mathcal{C}^{2}([0, a+b])$, but they not necessarily belong to $\mathcal{C}^{3}([0, a+b])$, which is physically consistent, due to the presence of the internal support at $x=a$. 
The following proposition contains an estimate of the third derivative of the eigenfunctions in each component $\Omega_{j}, j=1,2$.

Proposition 2 For every given number $M, M>1$, there exists a number $N_{M} \in \mathbb{N}, N_{M}$ only depending on $M$, such that

$$
\left\|S_{n}^{\prime \prime \prime}\right\|_{\mathrm{L}^{2}\left(\Omega_{j}\right)}^{2} \leq \lambda^{3 / 2} M\left\|S_{n}\right\|_{\mathrm{L}^{2}\left(\Omega_{j}\right)}^{2}, j=1,2, \quad \text { for every } n \geq N_{M} .
$$

Proof The proof is by direct computation. Let us consider $j=1$, the analysis for $j=2$ being analogous. From (3.18) and the normalization condition (3.14), for $x \in \Omega_{1}$ we have

$$
\begin{aligned}
S_{n}(x) & =\frac{1}{A_{n} \lambda_{n}}\left(\sin \left(\alpha_{n} x\right)-K_{\alpha_{n}, a} \sinh \left(\alpha_{n} x\right)\right), \\
S_{n}^{\prime}(x) & =\frac{\alpha_{n}}{A_{n} \lambda_{n}}\left(\cos \left(\alpha_{n} x\right)-K_{\alpha_{n}, a} \cosh \left(\alpha_{n} x\right)\right), \\
S_{n}^{\prime \prime \prime}(x) & =-\frac{\alpha_{n}^{3}}{A_{n} \lambda_{n}}\left(\cos \left(\alpha_{n} x\right)+K_{\alpha_{n}, a} \cosh \left(\alpha_{n} x\right)\right),
\end{aligned}
$$

where we have denoted $K_{\alpha_{n}, a}=\frac{\sin \left(\alpha_{n} a\right)}{\sinh \left(\alpha_{n} a\right)}$ and

$$
\left.A_{n}^{2}=\int_{\Omega_{1}}\left(\sin \left(\alpha_{n} x\right)-K_{\alpha_{n}, a} \sinh \left(\alpha_{n} x\right)\right)\right)^{2} \mathrm{~d} x
$$

Then

and

$$
\left(\frac{S_{n}^{\prime \prime \prime}(x)}{\alpha_{n}^{3}}\right)^{2}-\left(\frac{S_{n}^{\prime}(x)}{\alpha_{n}}\right)^{2}=\frac{2 K_{\alpha_{n}, a} \cos \left(\alpha_{n} x\right) \cosh \left(\alpha_{n} x\right)}{A_{n}^{2} \lambda_{n}^{2}}
$$

$$
\left\|S_{n}^{\prime \prime \prime}\right\|_{L^{2}\left(\Omega_{1}\right)}^{2}=\alpha_{n}^{2}\left\|S_{n}^{\prime}\right\|_{L^{2}\left(\Omega_{1}\right)}^{2}+\frac{2 \alpha_{n}^{6} K_{\alpha_{n}, a}}{A_{n}^{2} \lambda_{n}^{2}} \int_{\Omega_{1}} \cos \left(\alpha_{n} x\right) \cosh \left(\alpha_{n} x\right) \mathrm{d} x .
$$

A simple calculation shows that the sequence

$$
\left(K_{\alpha_{n}, a} \int_{\Omega_{1}} \cos \left(\alpha_{n} x\right) \cosh \left(\alpha_{n} x\right) \mathrm{d} x\right)_{n \in \mathbb{N}}
$$

is uniformly bounded. Since $\alpha_{n}=\lambda_{n}^{1 / 4}$, the last term in the right hand side of the above equation converges to zero as $n \rightarrow+\infty$ and, therefore, using also (3.16), for each $M>1$ there exists $N_{M} \in \mathbb{N}$ such that the desired estimated holds.

\subsection{Plate eigensolutions}

To solve (3.8)-(3.13) we use the Levy's method [12], assuming

$$
S(x, y)=\sum_{p \in \mathbb{N}} h_{p}(x) \sin \left(\frac{p \pi y}{L}\right) .
$$

The above expansion is justified by the fact that the family $\left\{\sin \left(\frac{p \pi y}{L}\right)\right\}_{p \in \mathbb{N}}$ is an Hilbert basis of $\mathrm{L}^{2}(0, L)$, with functions which satisfy the boundary conditions (3.9) at both the sides $y=0$ and $y=L$. Substituting (3.20) in (3.8) and using the orthogonality of the functions $\left\{\sin \left(\frac{p \pi y}{L}\right)\right\}_{p \in \mathbb{N}} \subset \mathrm{L}^{2}(0, L)$, we arrive at

$$
h_{p}^{\prime \prime \prime \prime}-2 \beta_{p} h_{p}^{\prime \prime}+\left(\beta_{p}^{2}-\lambda\right) h_{p}=0, \quad x \in(0, a) \cup(a, a+b),
$$


where $\beta_{p}=\frac{p^{2} \pi^{2}}{L^{2}}$.

To solve $(3.21)$, we first consider the case $\beta_{p}^{2} \neq \lambda$. We denote by $h_{p}^{a}$ and $h_{p}^{b}$ the solution of (3.21) in the intervals $(0, a)$ and $(a, a+b)$, respectively. The family of solutions of (3.21) defined in $(0, a)$ that satisfies the boundary conditions (3.9) on $x=0$ and the geometric condition (3.11) at $x=a$ is given by

$$
\begin{aligned}
h_{p}^{a, \sqrt{\lambda}}(x)=A_{a} & \left(-\frac{\sinh \left(\sqrt{\beta_{p}-\sqrt{\lambda}} a\right)}{\sinh \left(\sqrt{\beta_{p}+\sqrt{\lambda}} a\right)} \sinh \left(\sqrt{\beta_{p}+\sqrt{\lambda}} x\right)+\right. \\
& \left.+\sinh \left(\sqrt{\beta_{p}-\sqrt{\lambda}} x\right)\right)
\end{aligned}
$$

where $A_{a}$ is a non vanishing constant. Similarly, the solutions $h_{p}^{b, \sqrt{\lambda}}(x)$ of (3.21), defined in $(a, a+b)$ and satisfying the boundary conditions (3.9) on $x=a+b$ and the geometric condition (3.11) at $x=a$, can be simply deduced by the expression (3.22) by making the change of variables $\xi=a+b-x$, so that $\xi=0$ corresponds to $x=a+b$, and the interface between the two plates is located at $\xi=b$, or $x=a$. After imposing the jump conditions (3.12)-(3.13), we obtain, for each fixed $p \in \mathbb{N}$, the set of eigenvalues $\lambda_{p, q}, q \in \mathbb{N}$, as roots (different from $\beta_{p}^{2}$ ) of the resulting frequency equation

$$
\begin{aligned}
& \sqrt{\beta_{p}-\sqrt{\lambda_{p, q}}} \sinh \left(a \sqrt{\beta_{p}-\sqrt{\lambda_{p, q}}}\right) \cosh \left(b \sqrt{\beta_{p}-\sqrt{\lambda_{p, q}}}\right) \\
+ & \sinh \left(b \sqrt{\beta_{p}-\sqrt{\lambda_{p, q}}}\right)\left[\sqrt{\beta_{p}-\sqrt{\lambda_{p, q}}} \cosh \left(a \sqrt{\beta_{p}-\sqrt{\lambda_{p, q}}}\right)\right. \\
- & \sqrt{\sqrt{\lambda_{p, q}}+\beta_{p}} \sinh \left(a \sqrt{\beta_{p}-\sqrt{\lambda_{p, q}}}\right) \\
& \left.\left(\operatorname{coth}\left(a \sqrt{\sqrt{\lambda_{p, q}}+\beta_{p}}\right)+\operatorname{coth}\left(b \sqrt{\sqrt{\lambda_{p, q}}+\beta_{p}}\right)\right)\right]=0 .
\end{aligned}
$$

For each $p$, there exists a sequence $\left\{\lambda_{p, q}\right\}_{q \in \mathbb{N}}$ which gives a subset of the set of eigenvalues of problem (3.8)-(3.13), with $\lim _{q \rightarrow \infty} \lambda_{p, q}=\infty$. Since we are only interested in the asymptotic behavior of $\left\{\lambda_{p, q}\right\}_{q \in \mathbb{N}}$ for each $p$ fixed, dividing the above equation by $\sqrt{\beta_{p}-\sqrt{\lambda_{p, q}}}$ and taking the limit $q \rightarrow+\infty$ (that is $\lambda_{p, q} \rightarrow+\infty$ ), we obtain the asymptotic frequency equation

$$
\begin{aligned}
& \sin \left(a \sqrt{\sqrt{\lambda_{p, q}}-\beta_{p}}\right) \cos \left(b \sqrt{\sqrt{\lambda_{p, q}}-\beta_{p}}\right)+ \\
+ & \sin \left(b \sqrt{\sqrt{\lambda_{p, q}}-\beta_{p}}\right) \\
& \left(\cos \left(a \sqrt{\sqrt{\lambda_{p, q}}-\beta_{p}}\right)-2 \sin \left(a \sqrt{\sqrt{\lambda_{p, q}}-\beta_{p}}\right)\right)=0 .
\end{aligned}
$$

From (3.23), it is possible to conclude that, for each fixed $p \in \mathbb{N}$, the eigenvalues are such that $\lambda_{p, q}=\mathcal{O}\left(q^{4}\right)$ and, more importantly, the sequence $\left\{\sqrt{\lambda_{p, q}}\right\}_{q \in \mathbb{N}}$ is uniformly discrete. 
Finally, the eigenfunctions are given by

$$
S_{p, q}(x, y)= \begin{cases}h_{p}^{a, \sqrt{\lambda_{p, q}}}(x) \sin \left(\frac{p \pi y}{L}\right), & (x, y) \in[0, a] \times[0, L], \\ h_{p}^{b, \sqrt{\lambda_{p, q}}}(a+b-x) \sin \left(\frac{p \pi y}{L}\right), & (x, y) \in[a, a+b] \times[0, L] .\end{cases}
$$

When $\lambda=\beta_{p}^{2}$, the solutions of $(3.21)$ in the interval $(0, a)$ are

$$
h_{p}^{a, \sqrt{\lambda}}(x)=\frac{A\left(a \sinh \left(\sqrt{2 \beta_{p}} x\right)-x \sinh \left(\sqrt{2 \beta_{p}} a\right)\right)}{a \beta_{p}} .
$$

By proceeding in the same way as we did above, it is easy to show that there is only one $\beta^{2}(=\lambda)$ that corresponds to a not trivial solution of the two-span plate. We include this single value of $\lambda$ in the set $\left\{\lambda_{p, q}\right\}_{p, q \in \mathbb{N}}$ already obtained for the case $\beta^{2} \neq \lambda$, and for the sake of simplicity we continue to denote by $\left\{\lambda_{p, q}\right\}_{p, q \in \mathbb{N}}$ the set of eigenvalues of the problem. In particular, without loss of generality, the sequence of eigenvalues $\left\{\lambda_{p, q}\right\}_{p, q \in \mathbb{N}}$ is putted in increasingly order and, after the rearrangement, the sequence is denoted as $\left\{\lambda_{n}\right\}_{n \in \mathbb{N}}$, with $\lambda_{n}=\mathcal{O}\left(n^{4}\right)$ as $n \rightarrow \infty$.

\subsection{Series representation of the solution of the forced problem}

In this section we shall consider the forced vibration problems (2.1)-(2.8) and (2.9)-(2.15) for the two-span beam and plate, respectively. For the sake of simplicity, the case of the plate is analyzed in detail in the sequel. However, the analysis can be easily rephrased to deal with the beam problem.

We start with a representation result.

Lemma 1 Any element in $\mathrm{H}^{-1}\left(\Omega_{1} \cup \Omega_{2}\right)$ can be represented by $\sum_{n=1}^{+\infty} c_{n} \lambda_{n} S_{n}$ for some $\left\{c_{n}\right\}_{n \in \mathbb{N}} \subset \ell^{2}$.

Proof We first note that $X \subset \mathrm{H}_{0}^{1}\left(\Omega_{1} \cup \Omega_{2}\right)$. Then $\mathrm{H}^{-1}\left(\Omega_{1} \cup \Omega_{2}\right) \subset X^{\prime}$.

The set $\left\{S_{n}\right\} \subset X$ can be used to express any element in $\mathrm{H}^{-1}\left(\Omega_{1} \cup \Omega_{2}\right) \subset X^{\prime}$. In fact, let $F \in X^{\prime}$. By the Riesz Representation Theorem, there is an element $\sum_{n=1}^{+\infty} c_{n} S_{n} \in X$, with $\left\{c_{n}\right\}_{n \in \mathbb{N}} \subset \ell^{2}$ such that

$$
F(\phi)=\left\langle\phi, \sum_{n=1}^{+\infty} c_{n} S_{n}\right\rangle_{X}, \quad \forall \phi \in X .
$$

Integrating by parts and taking into account the boundary and jump conditions satisfied by the eigenfunctions $\left\{S_{n}\right\}$, we have

$$
\begin{aligned}
\left\langle\phi, \sum_{n=1}^{+\infty} c_{n} S_{n}\right\rangle_{X} & =\left\langle\phi, \sum_{n=1}^{+\infty} c_{n} S_{n}\right\rangle_{\mathrm{H}^{2}\left(\Omega_{1}\right)}+\left\langle\phi, \sum_{n=1}^{+\infty} c_{n} S_{n}\right\rangle_{\mathrm{H}^{2}\left(\Omega_{2}\right)} \\
& =\left\langle\triangle \phi, \triangle \sum_{n=1}^{+\infty} c_{n} S_{n}\right\rangle_{\mathrm{L}^{2}\left(\Omega_{1}\right)}+\left\langle\triangle \phi, \triangle \sum_{n=1}^{+\infty} c_{n} S_{n}\right\rangle_{\mathrm{L}^{2}\left(\Omega_{2}\right)} \\
& =\left\langle\phi, \sum_{n=1}^{+\infty} \lambda_{n} c_{n} S_{n}\right\rangle_{\mathrm{L}^{2}\left(\Omega_{1} \cup \Omega_{2}\right)}
\end{aligned}
$$


and the thesis follows.

The regularity of the solution to the forced vibration problem is considered in the next lemma.

Lemma 2 Let $w$ be the solution to (2.9)-(2.15) with $f \in \mathrm{H}^{-1}\left(\Omega_{1} \cup \Omega_{2}\right)$. Then $w \in$ $\mathcal{C}\left(\left[0, T_{0}\right], X\right) \cap \mathcal{C}^{1}\left(\left[0, T_{0}\right], \mathrm{L}^{2}\left(\Omega_{1} \cup \Omega_{2}\right)\right)$.

Proof To solve the nonhomogeneous problem we use the method of separation of time and spatial variables expanding the solution $w$ in eigenfunctions, namely

$$
w(x, y, t)=\sum_{m=1}^{\infty} \eta_{m}(t) S_{m}(x, y) .
$$

Following a standard procedure, we obtain a sequence of uncoupled linear second order differential equations on the unknowns $\left\{\eta_{m}(t)\right\}$ describing the forced motion of harmonic oscillators with natural frequency $\sqrt{\lambda_{m}}$. By Lemma 1 we have $f=\sum_{n=1}^{+\infty} c_{n} \lambda_{n} S_{n}$, with $\left\{c_{n}\right\}_{n \in \mathbb{N}} \subset \ell^{2}$, and then we obtain the following Duhamel representation formula

$$
w(x, y, t)=\sum_{m=1}^{+\infty} c_{m} \sqrt{\lambda_{m}} \int_{0}^{t} g(\tau) \sin \left(\sqrt{\lambda_{m}}(t-\tau)\right) \mathrm{d} \tau S_{m}(x, y) .
$$

Recalling that $g \in \mathcal{C}^{1}\left(\left[0, T_{0}\right]\right)$, by an integration by parts we see that there exists a constant $C_{T_{0}}>0$ independent of $\lambda_{m}$ such that

$$
\begin{aligned}
\int_{0}^{t} g(t-\tau) \sin \left(\tau \sqrt{\lambda_{m}}\right) \mathrm{d} \tau & \leq \frac{C_{T_{0}}}{\sqrt{\lambda_{m}}}\|g\|_{\mathcal{C}^{1}\left[0, T_{0}\right]}, \\
\frac{\partial}{\partial t} \int_{0}^{t} g(t-\tau) \sin \left(\tau \sqrt{\lambda_{m}}\right) \mathrm{d} \tau & \leq C_{T_{0}}\|g\|_{\mathcal{C}^{1}\left[0, T_{0}\right]} .
\end{aligned}
$$

From (3.24) and (3.25), we obtain that $u \in \mathcal{C}\left(\left[0, T_{0}\right], X\right)$, where the set $X$ has been defined in (3.17). Note that the traces of $w$ at the boundary are well defined. Finally, using (3.26), we can state that $w \in \mathcal{C}^{1}\left(\left[0, T_{0}\right], \mathrm{L}^{2}\left(\Omega_{1} \cup \Omega_{2}\right)\right)$, which means that the setting of the initial conditions makes sense.

\section{Proof of the uniqueness result}

We proceed to the proof of Theorem 2.1, dividing it in two parts to deal separately with beams and plates.

It is convenient to premise some definitions.

Definition 4.1 A sequence $\left\{\mu_{n}\right\}_{n \in \mathbb{N}} \subset \mathbb{C}$ is uniformly discrete if there exists $\delta>0$ such that $\left|\mu_{n}-\mu_{m}\right| \geq \delta$ for every $m, n \in \mathbb{N}$ with $m \neq n$.

Definition 4.2 Given a bounded set $U \subset \mathbb{R}^{d}, d \in \mathbb{N}$, with positive measure, the PaleyWiener space $P W_{S}$ is defined as

$$
P W_{U}=\left\{\hat{F} \mid F \in \mathrm{L}^{2}, \operatorname{supp}(F) \subset U\right\},
$$


where $\hat{F}$ is the Fourier transform of the function $F$.

In this paper we shall be interested only in the case when $d=1$.

Definition 4.3 The upper uniform density of a uniformly discrete set $\Lambda$ is defined by

$$
D(\Lambda)=\lim _{c \rightarrow+\infty} \max _{a \in \mathbb{R}} \frac{\#(\Lambda \cap(a, a+c))}{c} .
$$

Definition 4.4 An indexed set $\Lambda=\left\{\mu_{n}\right\}_{n \in \mathbb{N}} \subset \mathbb{R}$ is an interpolation set for $P W_{S}$, $S \subset \mathbb{R}$ bounded with positive measure, if for every sequence $\left\{d_{n}\right\}_{n \in \mathbb{N}} \subset \ell^{2}$ there exists $\phi \in P W_{S}$ such that $\phi\left(\mu_{n}\right)=d_{n}$ for every $\mu_{n} \in \Lambda$.

Finally, we recall that in the case of an interval $\left(a_{1}, a_{2}\right) \subset \mathbb{R}$, Kahane [13] (see also [14]) proved that

$$
\text { if } D(\Lambda)<\frac{1}{2 \pi}\left(a_{2}-a_{1}\right) \text {, then } \Lambda \text { is an interpolating set of } P W_{\left(a_{1}, a_{2}\right)} .
$$

Proof of Theorem 2.1 We interpret (3.24) as a distribution, and we make a change in the order of summation and integration, to obtain

$$
w(x, y, t)=\int_{0}^{t} g(t-\tau) \underbrace{\left[\sum_{m=1}^{+\infty} c_{m} \sqrt{\lambda_{m}} \sin \left(\sqrt{\lambda_{m}}(\tau)\right) S_{m}(x, y)\right]}_{\doteq F(\tau, x, y)} \mathrm{d} \tau .
$$

Using data (2.16) and Lemma 2, we can assert that for all $(t, x, y) \in[0, T] \times \Omega_{0}$,

$$
0=\frac{\partial w}{\partial t}(x, y, t)=g(0) F(t, x, y)+\int_{0}^{t} g^{\prime}(t-\tau) F(\tau, x, y) \mathrm{d} \tau .
$$

Since $g(0) \neq 0$ by hypothesis, we have necessarily that $F(t, x, y)=0$ for every $(t, x, y) \in$ $[0, T] \times \Omega_{0}$. It follows that

$$
F(t, x, y)=\sum_{m=1}^{+\infty} c_{m} \sqrt{\lambda_{m}} \sin \left(\sqrt{\lambda_{m}} t\right) S_{m}(x, y)=0, \forall(t, x, y) \in[0, T] \times \Omega_{0} .
$$

We can extend trivially $F$ to $[-T, T] \times \Omega_{0}$, because $t \mapsto \sin t$ is an odd function.

Now we split the proof in two parts to deal separately first with beams and, next, with plates.

For every $x \in \Omega_{0}$ and for every $\hat{\phi} \in \mathcal{C}_{c}^{\infty}((-T, T))$ we have

$$
\begin{aligned}
\langle F(\cdot, x), \hat{\phi}\rangle & =\langle\hat{F}(\cdot, x), \phi\rangle \\
& =\sum_{m=1}^{+\infty} c_{m} \sqrt{\lambda_{m}} S_{m}(x)\left(\phi\left(\sqrt{\lambda_{m}}\right)-\phi\left(-\sqrt{\lambda_{m}}\right)\right)=0 .
\end{aligned}
$$

Without loss of generality we can consider only odd functions $\phi$ in $P W_{(-T, T)}$ such that 
$\hat{\phi} \in \mathcal{C}_{c}^{\infty}\left(\left(-T_{0}, T_{0}\right)\right)$, to conclude that for any $x \in \Omega_{0}$ we have

$$
\sum_{m=1}^{+\infty} c_{m} \sqrt{\lambda_{m}} S_{m}(x) \phi\left(\sqrt{\lambda_{m}}\right)=0 .
$$

The sequence $\left(\sqrt{\lambda_{n}}\right)_{n \in \mathbb{N}}$ in the beam case is uniformly discrete, with $\sqrt{\lambda_{n}}=\mathcal{O}\left(n^{2}\right)$. Then, recalling that $\mathcal{C}_{c}^{\infty}((-T, T))$ is dense in $\mathrm{L}^{2}(-T, T)$, the result (4.1) can be applied with $D\left(\left\{\sqrt{\lambda_{n}}\right\}_{n \in \mathbb{N}}\right)=0$. It follows that $c_{m} S_{m}(x)=0$ for every $m \in \mathbb{N}$. Since $\left\{S_{m}\right\}_{m \in \mathbb{N}}$ forms a complete Hilbert base, we conclude that $c_{m}=0$ for every $m \in \mathbb{N}$.

In the case of plates, we test the analogous expression of (4.2) also with respect to space variables to obtain

$$
\sum_{p=1}^{+\infty} \sum_{q=1}^{+\infty} c_{p, q} \sqrt{\lambda_{p, q}}\left\langle S_{p, q}, \varphi\right\rangle \phi\left(\sqrt{\lambda_{p, q}}\right)=0, \forall \phi \in P W_{(-T, T)} \text { and } \forall \in \mathcal{C}_{c}^{\infty}\left(\Omega_{0}\right) .
$$

Now, recalling the explicit form of the eigenfunctions $S_{p, q}$ obtained in Section 3.3 and since $\Omega_{0}$ contains the line $\left\{\left(x_{0}, y\right): y \in[0, L]\right\}$, we see that given $p=p_{0} \in \mathbb{N}$ it is always possible to choose $\varphi \in \mathcal{C}_{c}^{\infty}\left(\Omega_{0}\right)$ so that $\left\langle S_{p, q}, \varphi\right\rangle=0$ if $p \neq p_{0}$ and $\left\langle S_{p, q}, \varphi\right\rangle \neq 0$ otherwise. Therefore, for every $p_{0} \in \mathbb{N}$ we get

$$
\sum_{q=1}^{+\infty} c_{p_{0}, q} \sqrt{\lambda_{p_{0}, q}}\left\langle S_{p_{0}, q}, \varphi\right\rangle \phi\left(\sqrt{\lambda_{p_{0}, q}}\right)=0, \forall \phi \in P W_{(-T, T)} \text { and } \forall \varphi \in \mathcal{C}_{c}^{\infty}\left(\Omega_{0}\right) \text {. }
$$

Now we recall that the sequence $\left\{\sqrt{\lambda_{p_{0}, q}}\right\}_{q \in \mathbb{N}}$ is uniformly discrete, with $\sqrt{\lambda_{p_{0}, q}}=\mathcal{O}\left(q^{2}\right)$. It follows that, exactly as in the beam case, $c_{p_{0}, q}=0$ for every $p_{0}, q \in \mathbb{N}$.

In conclusion, for both beam and plate multi-span systems, the loading $f \in H^{-1}\left(\Omega_{1} \cup\right.$ $\left.\Omega_{2}\right)$ is null, and therefore the spatial component of the forcing source in (2.1)-(2.8) and (2.9)-(2.15) can be uniquely identified by the data specified in the set $\Gamma$.

\section{Numerical experiment}

In this section we provide a numerical experiment to illustrate the practical possibility of identifying sources in multi-span systems. The numerical experiment is focussed on the two-span beam described in (2.1)-(2.8) and, adopting the same notation used above, the length parameters are $a=1$ and $b=2.5$. In this case, the first ten eigenvalues are $\left\{\lambda_{n}\right\}_{n=1}^{10}=\left\{4.33,4.7 \times 10^{1}, 1.43 \times 10^{2}, 2.68 \times 10^{2}, 7.19 \times 10^{2}, 1.56 \times 10^{3}, 1.14 \times 10^{4}, 1.73 \times\right.$ $\left.10^{4}, 2.49 \times 10^{4}, 7.62 \times 10^{4}\right\}$. The eigenfunctions associated to the first six eigenvalues are shown in Figure 3.

The time-dependent function $g=g(t)$ is chosen as $g(t)=\cos (t)$, whereas the function $f=f(x)$ that describes the spatial distribution of the loading is taken coincident with the characteristic function of the interval $[a+b / 3, a+2 b / 3]$.

It should be noticed that in all the calculations performed to obtain the solution of the direct problem, the function $f=f(x)$ is represented by the truncated series expansion $\widetilde{f}(x)=\sum_{n=1}^{100} c_{n} \lambda_{n} S_{n}(x)$. Figure 4 compares the target function $f(x)$ with the approximation $\tilde{f}(x)$ really used to perform the calculations of this section.

The solution of the direct problem is given by (3.24). For this concrete example, we show in Figure 5(a) the displacement at time $t=2$ of the whole continuous beam 

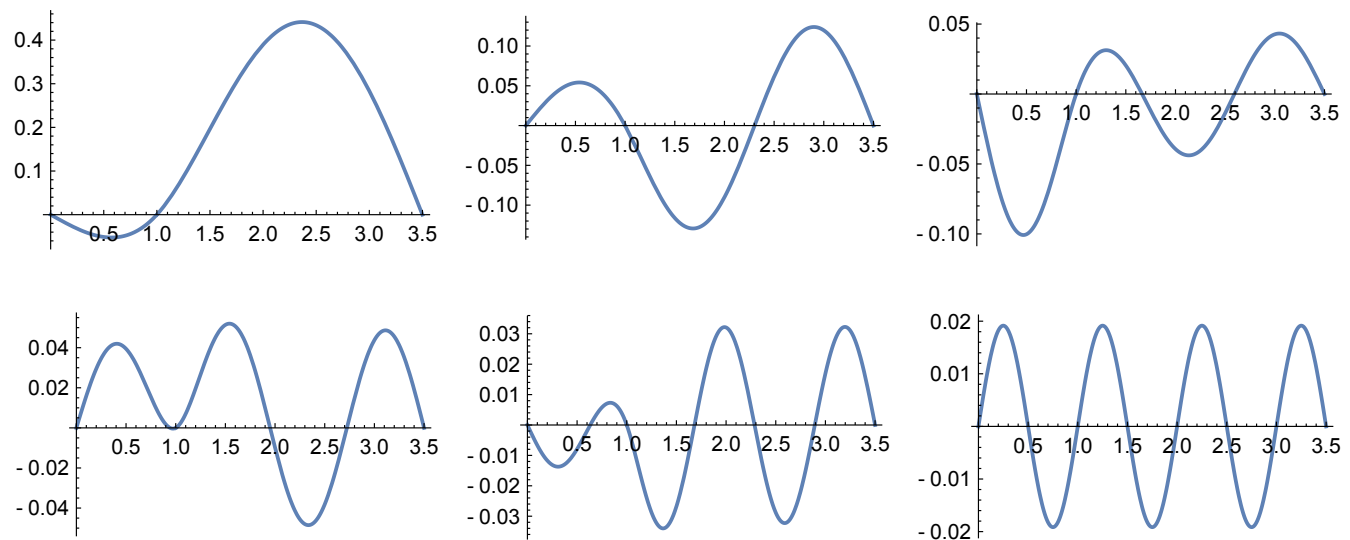

Figure 3. First six (normalized) eigenfunctions of the problem (2.1)-(2.8) with $a=1$ and $b=2.5$.

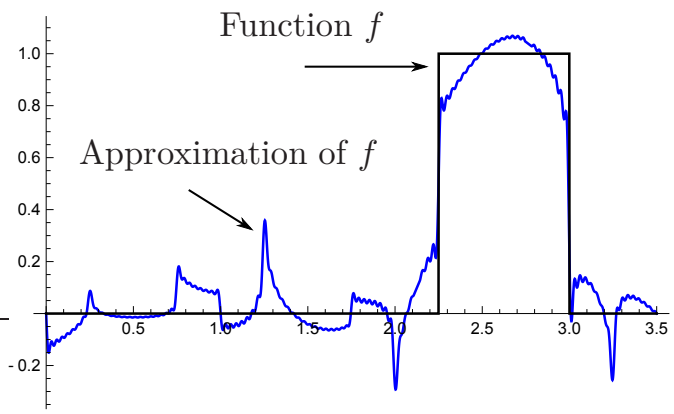

FiguRE 4. Function $f=f(x)$ (black line) and its approximation $\tilde{f}(x)$ (gray line) used in the computations.

(that is, $x \mapsto u(x, 2)$ ). For the solution of the inverse problem, the measurement of the displacement is taken at $x_{0}=0.77$. In Figure $5(\mathrm{~b})$ we show the displacement at point of measurement $x_{0}=0.77$ in the time interval $t \in[0,50]$.

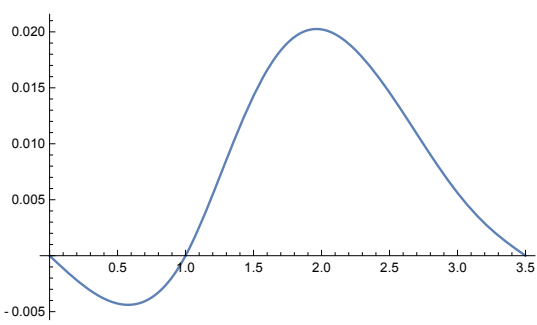

(a) Displacement $u=u(x, t)$ over the whole structure for $t=2$.

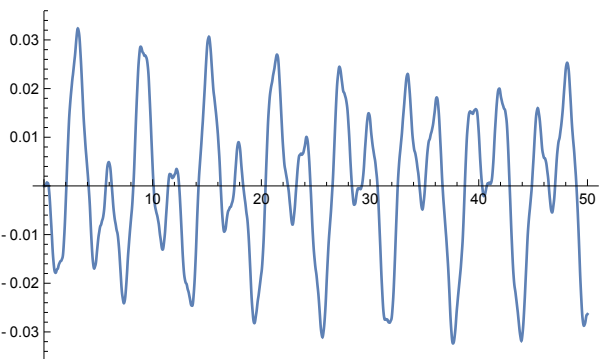

(b) Displacement $u\left(x_{0}, t\right)$ at point $x_{0}=0.77$ in the time interval $t \in[0,50]$.

FIGURE 5. Solution $u(x, t)$ of the direct problem obtained by (3.24) using the approximation $\widetilde{f}(x)$ instead of the function $f=f(x)$.

Since it is not our main objective to discuss a numerical procedure to recover the 
spatial loading $f$ described by the coefficients $\left\{c_{n}\right\}_{n \in \mathbb{N}}$, we just present succinctly the procedure that is described in detail in [15].

Following [15], we use the family of test functions

$$
\phi_{m, \tau}(\xi)=\left(\frac{\sin \left(\left(\xi-\lambda_{m}\right) \tau\right)}{\left(\xi-\lambda_{m}\right) \tau}\right)^{2}, \quad \forall m \in \mathbb{N} \text { and } \forall \tau>0 .
$$

It is important to notice that their Fourier transform have compact supports, namely

$$
\widehat{\phi_{m, \tau}}(t)=\left(H_{\tau} * H_{\tau}\right)(t) e^{-i t \lambda_{m}}, \quad H_{\tau}(t)=\frac{1}{2 \tau} \chi_{(-\tau, \tau)}(t),
$$

and this means that the measurements are performed in finite intervals of time.

With the particular time function $g$ chosen in this numerical simulation, the displacement function at point $x_{0}, u\left(t, x_{0}\right)$, given in (3.24) (with $w$ replaced by $u$ ) can be rewritten as

$$
u\left(t, x_{0}\right)=\sum_{m=0}^{+\infty} B_{m}\left(\mathrm{e}^{i \sqrt{\lambda_{m}} t}+\mathrm{e}^{-i \sqrt{\lambda_{m}} t}\right),
$$

where $\left\{\lambda_{n}\right\}_{n \in \mathbb{N}}$ is given by the procedure described in Section 3.2 and $\lambda_{0}=1$, e.g., $\lambda_{0}$ accounts for the function $g(t)=\cos (t)$. For $m \geq 1, B_{m}$ is defined by

$$
B_{m}=\frac{c_{m} \lambda_{m}}{\lambda_{m}-1} S_{m}\left(x_{0}\right)
$$

whereas $B_{0} \doteq \sum_{m=1}^{+\infty} B_{m}$.

In order to apply the procedure described in [15], we must reorder the sequence $\left\{\lambda_{m}\right\}_{m \in \mathbb{N}} \cup\left\{-\lambda_{m}\right\}_{m \in \mathbb{N}}$ so that $\lambda_{0}$ is the first term, $-\lambda_{0}$ the second one, $\lambda_{1}$ the third one, $-\lambda_{1}$ the forth one, and so on. The ordered set of $\lambda$ s becomes then

$$
\left\{\lambda_{0},-\lambda_{0}, \lambda_{1},-\lambda_{1}, \lambda_{2},-\lambda_{2}, \lambda_{3},-\lambda_{3}, \ldots\right\} .
$$

Realizing that the same $B_{m}$ corresponds to both $\lambda_{m}$ and $-\lambda_{m}$ in (5.2), the ordered set of $B \mathrm{~s}$, counting repetitions, becomes

$$
\left\{B_{0}, B_{0}, B_{1}, B_{1}, B_{2}, B_{2}, B_{3}, B_{3}, \ldots\right\} \text {. }
$$

We perform this rearrangement of terms, but we keep the same index $m$. We define $P_{\tau}(m, n)=\phi_{m, \tau}\left(\lambda_{n}\right)$ and

$$
V(m)=\left\langle u\left(\cdot, x_{0}\right), \widehat{\phi_{m, \tau}}\right\rangle .
$$

Consider the operator $T: \ell^{2} \rightarrow s^{\prime}$, given $\left(B_{m}\right)_{n \in \mathbb{Z}_{+}} \rightarrow(V(m))_{m \in \mathbb{Z}_{+}}$. Formally, the application of the operator $T$ can be interpreted as performing a product with a matrix of infinite order, that is

$$
\left[\begin{array}{c}
V(0) \\
V(1) \\
\vdots
\end{array}\right]=\left[\begin{array}{cccc}
P_{\tau}(0,0) & P_{\tau}(0,1) & P_{\tau}(0,2) & \ldots \\
P_{\tau}(1,0) & P_{\tau}(1,1) & P_{\tau}(1,2) & \ldots \\
\vdots & \vdots & \vdots & \ldots
\end{array}\right]\left[\begin{array}{c}
B_{0} \\
B_{1} \\
\vdots
\end{array}\right]
$$


We perform now a truncation of the system in (5.4) to obtain

$$
\left[\begin{array}{c}
V(0) \\
V(1) \\
\vdots \\
V(N)
\end{array}\right]=\underbrace{\left[\begin{array}{cccc}
P_{\tau}(0,0) & P_{\tau}(0,1) & \ldots & P_{\tau}(0, N) \\
P_{\tau}(1,0) & P_{\tau}(1,1) & \ldots & P_{\tau}(1, N) \\
\vdots & \vdots & \vdots & \vdots \\
P_{\tau}(N, 0) & P_{\tau}(N, 1) & \ldots & P_{\tau}(N, N)
\end{array}\right]}_{\mathrm{T}_{\mathrm{N}}}\left[\begin{array}{c}
B_{0} \\
B_{1} \\
\vdots \\
B_{N}
\end{array}\right] .
$$

By solving the linear system (5.5), with symmetric $N \times N$ matrix $T_{N}$, we obtain the first elements of the desired sequence $\left\{B_{m}\right\}_{n \in \mathbb{Z}_{+}}$, and using (5.3), we recover the first elements of the sequence $\left\{c_{m}\right\}_{m \in \mathbb{N}}$ that defines the function $f$. In fact, performing the calculations assuming $x_{0}=0.77$, length of registration time $T=5$, and evaluating the solution of the direct problem by (3.24) with the approximation $\widetilde{f}(x)$ of the actual force $f(x)$, we recovered the first $N=14$ terms of the sequence $\left\{c_{m}\right\}_{m \in \mathbb{N}}$. With these recovered terms, the reconstructed function is shown in Figure 6. Note the good match between the recovered function and the function used in the calculations of the direct problem (see also Figure 4).

Since it was not our objective in this article to analyze the effects of measurement errors, but to show a proof of the uniqueness Theorem 2.1, we did not introduced measurement errors in the simulation.

However, for the sake of completeness in our discussion, we observe that when the measurements contain errors, then instead of $V(m)$, we have at our disposal $\tilde{V}(m)=$ $\left\langle\tilde{u}\left(t, x_{0}\right), \widehat{\phi_{1, m, \tau}}\right\rangle$, where the difference between $\tilde{u}\left(t, x_{0}\right)$ and $u\left(t, x_{0}\right)$ is the error $e(t) \doteq$ $\tilde{u}\left(t, x_{0}\right)-u\left(t, x_{0}\right)$.

In this case, the solution of the linear system (5.5) gives

$$
\left(\tilde{B}_{n}\right)_{n=1}^{N}=\mathrm{T}_{\mathrm{N}}^{-1}\left((V(m))_{m=1}^{N}\right)+\mathrm{T}_{\mathrm{N}}^{-1}\left(\left(V_{e}(m)\right)_{m=1}^{N}\right),
$$

where

$$
V_{e}(m)=\left\langle e, \widehat{\phi_{1, m, \tau}}\right\rangle .
$$

In [15] it is proved that $\mathrm{T}_{\mathrm{N}}^{-1}\left((V(m))_{m=1}^{N}\right) \stackrel{N \rightarrow+\infty}{\longrightarrow}\left(\left(\alpha_{n}\right)_{n \in \mathbb{N}}\right)$ and that when the error $e(t)=\tilde{u}\left(t, x_{0}\right)-u\left(t, x_{0}\right)$ is bounded in $\mathrm{L}^{2}\left(0, T_{0}\right)$ by $\|e\|_{\mathrm{L}^{2}(0, \tau)} \leq \epsilon$, then there exists $C>0$ such that $\left.\| \mathrm{T}_{\mathrm{N}}{ }^{-1}\left(V_{e}(m)\right)\right)_{m=1}^{N} \|$ is given by

$$
\left.\| \mathrm{T}_{\mathrm{N}}{ }^{-1}\left(V_{e}(m)\right)\right)_{m=1}^{N}\|\|_{\ell^{2}} \leq C \epsilon \sqrt{\frac{N+1}{2 \tau}} .
$$

In our actual calculations, the match between the recovered function and the approximation of $f$, which was used in all calculations of the direct problem (obtained by a truncation of the series development $\sum_{n=1}^{+\infty} c_{n} \lambda_{n} S_{n}$ with 100 terms) is not exact because in the recovery process we used only 14 terms. In fact, when the number of terms used in both direct and inverse problems is equal, then the match is perfect, but then an inverse crime is perpetrated.

The whole formal numerical analysis performed above, including convergence and stability considerations, can be rigorously discussed by adapting the arguments shown in $[15]$. 


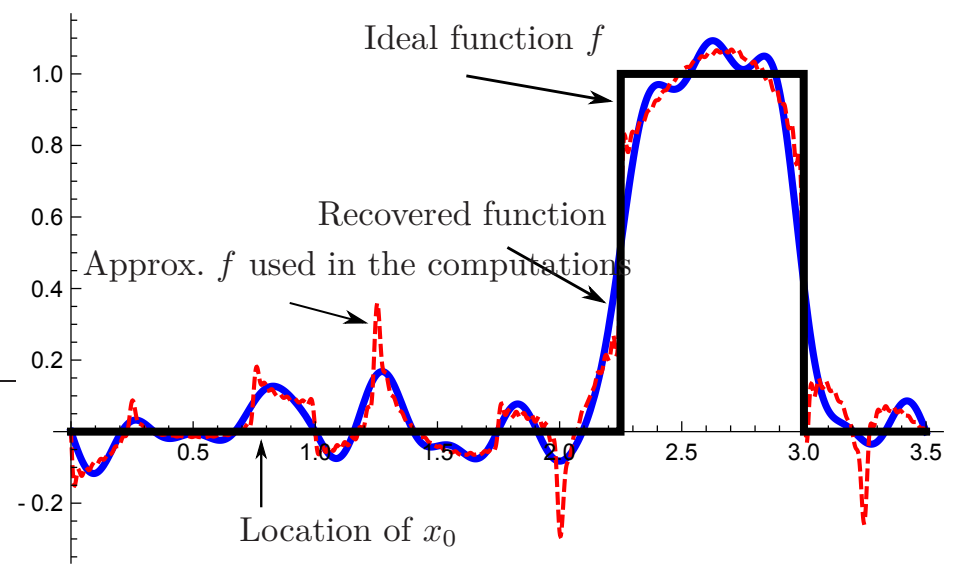

FiguRE 6. An example of reconstruction of the unknown function $f$ for the two-span beam system (2.1)-(2.8).

\section{Conclusion}

Despite their practical importance, inverse problems involving multi-span structures are not discussed in the literature, to our knowledge. As a contribution to the field of inverse problems related to structural engineering, in this article we have proved a Theorem (Theorem 2.1) that essentially states that the spatial distribution of loads of of the form $g(t) f(x)$, with $g \in \mathcal{C}^{1}$ known, can be uniquely identified given the information about the displacement of a neighborhood of a) a point in the interior of the structure in the case of beams, or b) the displacement of a line in the case of plates. In both cases the observation interval of time can be arbitrarily small. It is important to note that even if the spatial support of the load is on a span and the measurement is at another span of the structure, the identification is still possible. This result enables a rigorous discussion about the identifiability of vibration sources in complex structures.

\section{Acknowledgements}

A. Kawano acknowledges the support provided by CNPq Proc. 304972/2013-4 and Fapesp Proc. 2015/50461-0. A. Morassi gratefully acknowledges the financial support of the National Research Project PRIN 2015TTJN95 "Identification and monitoring of complex structural systems".

\section{References}

[1] Ray W. Clough and Joseph Penzien. Dynamics of Structures. Computers \& Structures, Inc, 3rd. edition, 1995.

[2] Owen F. Hughes. Ship Structural Design. John Wiley \& Sons, 3rd. edition, 1995.

[3] Alemdar Hasanov and Alexandre Kawano. Identification of an unknown spatial load distribution in a vibrating Euler-Bernoulli beam from limited measured data. Inverse Problems, 32(5):055004, 2016.

[4] Yamamoto M. Determination of forces in vibrations of beams and plates by pointwise and line observations. Journal of Inverse and Ill Posed Problems, 4(5):437-457, 1996. 
[5] Kawano A. Uniqueness results in the identification of distributed sources over Germain-Lagrange plates by boundary measurements. Applicable Analysis, 95(9):2004-2016, 2015.

[6] Nicaise S. and Zaïr O. Determination of point sources in vibrating beams by boundary measurements: Identifiability, stability, and reconstruction results. Electronic Journal of Differential Equations, 2004(20):1-17, 2004.

[7] Zaïr O. Determination of point sources in vibrating plate by boundary measurements. Applicable Analysis, 92(10):2061-2075, 2013.

[8] Wang Y.H. Global uniqueness and stability for an inverse plate problem. Journal of Optimization Theory and Applications, 132(1):161-173, 2007.

[9] Alves C., Silvestre A.L., Takahashi T., and Tucsnak M. Solving inverse source problems using observability. Applications to the Euler-Bernoulli plate equation. SIAM Journal on Control and Optimization, 48(3):1632-1659, 2009.

[10] Nicaise S. and Zaïr O. Identifiability, stability and reconstruction results of point sources by boundary measurements in heteregeneous trees. Revista Matemática Complutense, 16(1):151-178, 2003.

[11] Kawano A. Uniqueness in the determination of vibration sources in rectangular Germain-Lagrange plates using displacement measurements over line segments with arbitrary small length. Inverse Problems, 29(8):085002, 2013.

[12] Timoshenko S.P. and Woinowsky-Krieger S. Theory of Plates and Shells. McGraw-Hill, 2nd edition, 1970.

[13] Kahane J.-P. Sur les fonctions moyenne-périodiques bornées. Annales de l'Institut Fourier, 7:293-314, 1957.

[14] Olevskii A. and Ulanovskii A. Interpolation in Bernstein and Paley-Wiener spaces. Journal of Functional Analysis, 256(10):3257-3278, 2009.

[15] Kawano A. and Zine A. Uniqueness and nonuniqueness results for a certain class of almost periodic distributions. SIAM Journal of Mathematical Analysis, 43(1):135-152, 2011. 